\title{
AUTOEVALUACIÓN, AUTORREGULACIÓN Y ACREDITACIÓN: RETOS DE LA EDUCACIÓN SUPERIOR
}

Olga Maria Villalta

\footnotetext{
Resumen: El articulo presenta una revisión amplia sobre lo que reconocidos especialistas principalmente de América. plantean referente a la autoevaluación, autoTegulación y acreditación de la Educación Superior. El prapasito es aportar información especializada con la pretención de que genere inquietudes y contribuya con la reflexión que debe originarse respecto a este tema, en las instituciones de Educación Superior del país.

En las unitersidades latinoamericanas se escucha cada tez con mayor frecuencia la necesidad de bacer frente a procesos de eutoevaluación, autorregulación y acreditación.

Los autores revisados asumen posiciones optimistas al respecto, consideran que el carácter voluntario y participativo de las propuestas, posibilita a las instituciones a generar respuestas prospectivas más importantes aün en tiempos de crisis económica y social.

Sin embargo, casi la totalidad de los documentos revisados son de carácter conceptual y técnico; carecen de análisis critico y no aluden a variables centrales en el proceso educativo como son los fundamentos ético-pistemológicos y enfoques pedagógicos.
}

\section{Presentación}

Al tomar en cuenta que actualmente la Educación Superior Estatal Costarricense, propone a la comunidad universitaria el incorporarse decididamente en procesos de autoevaluación y autorregulación para la acreditación, se consideró oportuno ofrecer una revisión sobre lo que diferentes autores proponen en relación con estos procesos y sus vinculaciones.

El propósito es aportar material de interés con la aspiración de que genere inquietudes y sea útil para la reflexión y discusión de las instituciones y/o programas que deben interrogarse sobre estos procesos. Cuando es posible, se incorporan datos sobre experiencias de países latinoamericanos; especialmente sucede esto en relación con los procesos de regulación en Chile.

Antes de desarrollar el tema, y teniéndose claro que no es objetivo de éste, artículo oportuno destacar la necesidad de insertarse en estos procesos de manera crítica e inteligente ${ }^{1}$. Al respecto Gurdián Fernández, A. señala "Nuestro peor error sería concebir la acreditación universitaria como algo ahistórico, descontextualizado, políticamente neutro y totalmente reactivo". (Gurdián 1997: 84).

Dentro de esta perspectiva es necesario recordar la importancia de considerar los fundamentos ético-epistemológicos que sustentan los procesos de autoevaluación y autorregulación para la acreditación. Según propuesta de Masís, Carlos, los fundamentos 
ético-epistemológicos deben constituirse en la base de una pirámide que culmina con el proceso de acreditación.

Además de lo anterior, Castillo y otros señalan que todo juicio referido a procesos educativos, indispensablemente debe darse en el marco de un esfuerzo de interpretación del sentido de los hechos. (Castillo, 1996: 10).

El artículo se organiza de la siguiente manera; inicialmente se anotan aspectos de carácter introductorio; posteriormente se definen y caracterizan cada uno de los procesos señalados: autoevaluación, autorregulación y acreditación; también se identifican y analizan sus interacciones. Finalmente se incorporan algunas reflexiones a modo de conclusiones generales.

\section{Aspectos introductorios}

En tiempos de crisis las universidades públicas también son blanco de ajustes financieros. Recuérdese la óptica neoliberal de focalizar los recursos del Estado en los niveles básicos: por ejemplo en Costa Rica, las actuales políticas educativas se dirigen a reforzar principalmente, los niveles de enseñanza preescolar y enseñanza primaria lo que podría traer como consecuencia reducir el apoyo financiero a la Educación Superior.

De ahí la necesidad de que las instituciones de Educación Superior Pública generen procesos permanentes de autorregulación-autoevaluación (Kells, 1997 y Lemaitre s.f) que les permita ser prospectivos y en consecuencia adelantarse a probables regulaciones emanadas de los gobiernos ó en el nivel interno institucional, adelantarse a regulaciones venidas de la dirigencia universitaria. A su vez ambos procesos (autoevaluación-autorregulación) permiten o facilitan el acceder a la acreditación: reconocimiento público de la calidad del servicio educativo.

En los últimos años en los países latinoamericanos se escuchan cada vez con más énfasis los temas de autorregulación, autoevaluación y acreditación. La autorregulación concretamente, es un concepto primordial directamente relacionado con las aspiraciones y características operativas de desarrollo de una institución. Una organización de educación superior autorregulada se adelanta a regulaciones externas, moviliza sus recursos especialmente a los humanos, para responder a los retos que afronta y no espera a recibir instrucciones y ser sujeta a limitaciones por parte del gobierno ${ }^{2}$ (Kells, 1997:44).

Por otro lado, en América Latina, los procesos anteriores también constituyen un buen modo de enfrentar competencias "desleales" de Educación Superior Privada que proliferan en los últimos tiempos, sin presentar la mayoría de las veces los niveles de excelencia requeridos.

Los conceptos de autoevaluación, autorregulación y acreditación en la Educación Superior tienen procedencia norteamericana (Estados Unidos). Desde los años 50's y 60's algunas universidades de ese país ya realizaban revisiones periódicas como parte del sistema de acreditación de instituciones y programas académicos.

Estos conceptos traducen, de algún modo, a los profesionales de la Educación Superior contenidos procedentes del desarrollo organizacional y del movimiento de gestión de la calidad total (TQM, Total Quality Management). Estos últimos conceptos se generaron en el ámbito de la industria y del gobierno principalmente, desarrollando conceptos ampliamente difundidos como son: "rediseño", "reingeniería", "reinversión", "mejoramiento continuo". Todos ellos prácticamente sinónimos de una autoevaluación basada en la cibernética y orientada a los requerimientos del cliente. Muchos académicos de la Educación Superior cuestionan estas propuestas, específicamente en relación al TQM opinan que no obstante su intención de ser holístico (un abordaje acerca de la calidad a través de toda la institución) opinan que sus simbolismos grupales no se adaptan a las subculturas profesionalmente anárquicas, multidisciplinarias y de otro tipo de universidades. Considera (Kells, H., Ibid 40) que pa- 
ra llegar a ser efectivo, es necesario que los conceptos de gestión de la calidad total deben ir acompañados de las prácticas familiares (idiosincracia) de la institución.

En América Latina las instituciones de Educación Superior carecen de lo que se ha dado en denominar "cultura de autoevaluación" y cuando este esfuerzo se hace (titánico para sus hacedores) no ha generado mayores dividendos: adolecen de esfuerzos inútiles y hasta de duplicaciones frecuentes.

Para efectos de ahondar en los procesos anteriores se revisarán separadamente cada uno de los conceptos: autoevaluación, autorregulación, acreditación, teniéndose en cuenta su estrecha articulación particularmente de los primeros. Ambos, autoevaluación y autorregulación proveen la información y condiciones para que se dé la acreditación.

Es descle este punto de vista, según la autora, que la autoevaluación, autorregulación y acreditación constituyen retos para nuestras universidades públicas: se constituyan procesos críticos que estén determinados y conducidos por las mismas universidades y enmarcados en los contextos económicos, sociales, culturales y políticos en que están inmersos.

\section{La Autoevaluación en la Educaciōn Superior}

\section{1 ¿Qué es la Autoevaluación?}

Según, (Silva, T. M., s.f), de la Universidad de Concepción de Chile: la autoevaluación institucional es un proceso de estudio global de la institución o de una de las áreas, de carácter esencialmente analítico, reflexivo $y$ orientado al cambio que es organizado y conducido de manera participativa por directivos y académicos a la luz del contexto institucional y criterios estándares. Además del propósito central de mejorar el nivel de desempeño, otros beneficios del proceso de autoestudio, son: (Kells, 1997:58). a. La participación significativa de los miembros en el proceso de análisis resultan frecuentemente en una identificación más positiva con la organización y visualización se sienten comprometidos a llevarlos a cabo. Lo que en desarrollo organizacional "se ha dado en llamar cemento psicológico". En otras palabras reducir la brecha existente muchas veces entre las metas personales y las organizacionales.

b. Fomento de la apertura institucional: mejorar patrones de comunicación conforme al funcionamiento de grupos. Ello constituye un potencial para realizar la eficacia de la organización. "Si son tratadas como miembros valiosos y de confianza de la comunidad y si se puede extraer de ellas sus mejores esfuerzos para lograr las metas organizacionales y personales, entonces se verá realizada la eficacia de la organización".

c. Mejorar en la eficacia global de la institución o programas.

d. Medio importante para el desarrollo del personal: pueden surgir nuevos líderes comprometidos.

e. Puede también estimular la revisión de políticas largamente descuidadas, de prácticas, de procedimientos, de archivos y dar lugar a innovaciones útiles en los programas.

f. Al generar un proceso analítico-participativo puede constituirse en una presentación útil de la organización, particularmente para los miembros más nuevos.

Víctor Cruz Cardona en su definición de autoevaluación enfatiza en lo concerniente a la participación y sus propósitos. La autoevaluación es un proceso que como su nombre lo indica, lo planifican, lo organizan, lo ejecutan y lo supervisan todas aquellas personas comprometidas con el programa o con la institución objeto de evaluación ...... y persigue dos propósitos centrales: identificar 
los problemas, las dificultades, los aciertos y los logros de un programa y proponer, introducir y comprometerse con revisiones y ajustes que permitan mejorarlo cualitativamente.

\subsection{El contexto de la Autoevaluación}

La autoevaluación es un proceso que se ubica al interior de una organización social. La atomización del mundo occidental ha conducido a pensar lo gerencial como vinculado solo a lo empresarial y económico y no a lo académico, lo cual obviamente no es cierto. La institución de educación superior constituye una organización social objeto de gerencia (obviamente con características muy particulares) que posee diferentes niveles.

Por tanto la autoevaluación es un elemento presente en cada uno de esos niveles: personas, programas y servicios. Los procesos de autoevaluación forman parte de las subfunciones secuenciales del proceso de gerencia de una institución o unidad académica. De ahí la necesidad de que los académicos conozcan el entorno organizacional y particularmente las características "anti-administrativas" de la academia. "En general las instituciones de educación superior no suelen exhibir una gestión vívida. Tienden a reaccionar en lugar de actuar: el cambio ocurre lentamente" (Kells, 1997:23).

Véase el siguiente esquema de un proceso de autoevaluación orientado al cambio.

Un proceso de autoevaluación orientado al mejoramiento que haga posible que los participantes asuman la propiedad psicológica de las propuestas para el cambio al interior de conclusiones de confianza; incentivos, asistencia técnica, liderazgo efectivo y opciones en el diseño (qué estudiar, cómo y en qué secuencia).

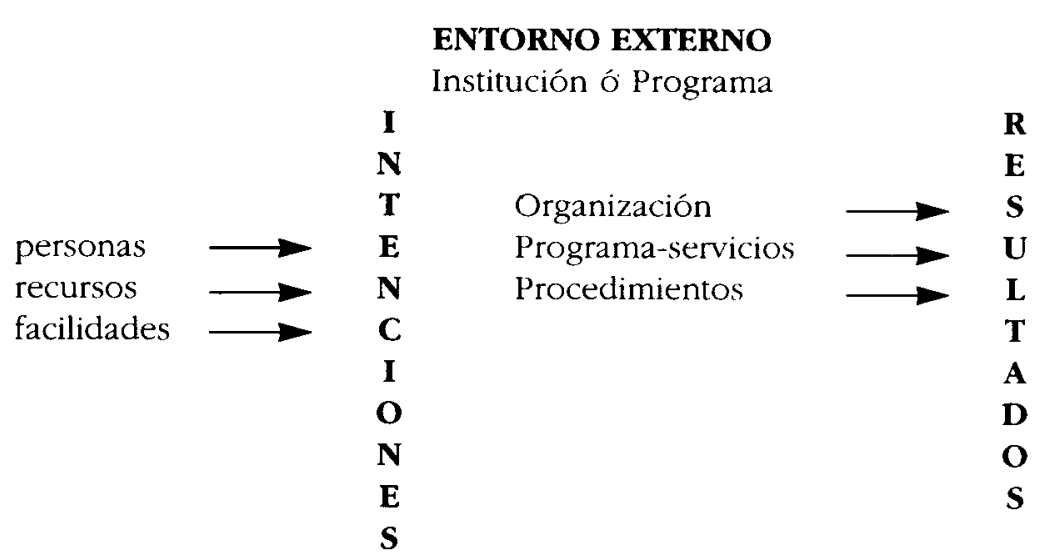

ENTORNO EXTERNO

La definición operativa de un proceso de autoevaluación orientado al mejoramiento, adoptado de (Kells: 1997:59).
Recomendaciones para lograr:

- mejoras en los insumos, las condiciones, los programas, los servicios, los procedimientos y los resultados,

- garantía de una adecuada calidad de los insumos, contenidos, procesos, servicios y productos finales (D-I-AS), promoción de cultura e infraestructura para la autoevaluación y la autorregulación. 


\subsection{Relación: autoevaluación- autorregulación}

(J. Alirio Ortiz R., 1994:51) plantea una concepción de autoevaluación plenamente vinculada, o más aún como parte del proceso de autorregulación; manifiesta "el elemento esencial que permitirá a una institución sobrevivir y competir con estándares con excelencia de calidad, es el mecanismo autorregulador dinámico indagante que es la autoevaluación" (Ortiz, 1994:51). Evidentemente el autor destaca el papel de mecanismo de control de la autoevaluación. Insta a recordar que toda institución docente es realmente una empresa y por lo mismo la autoevaluación participa de los fines de ella: cuida de que sus planes se cumplan y de distribuir económicamente la utilización de sus recursos. En este sentido, continúa manifestando (Ortiz R., Ibid:58) "la evaluación es homologada al mecanismo de control que como tal, se enfoca a conocer los resultados de todas las acciones de la empresa, utilizando el medio de retroalimentación según la concepción sistémica en boga".

\subsection{Relación: autoevaluación y acreditación}

Se hace oportuno destacar la importancia de no supeditar el proceso de autoevaluación a la acreditación: al respecto se señala que las organizaciones académicas existen porque tienen una misión social de enorme trascendencia y esto debe ser objeto de una reflexión periódica y organizada (autoevaluación). (Ortiz R., 1995:4).

Es en referencia a esto, continúa ese autor, que deben automirarse y no en referencia a un requerimiento externo que tiende a condicionar dadas sus consecuencias: un juicio, una calificación, una certificación, etc. En otras palabras la institución de educación superior no existe para acreditarse: esta es externa al quehacer y compromiso social de la institución. Más adelante el mismo autor manifiesta:
"Sin duda alguna al estar condicionada la autoevaluación a la acreditación, el esfuerzo puede orientarse muy posiblemente a presentar una imagen más ficticia que real por cuanto ello favorece a los intereses pretendidos de obtener un beneplácito posterior" (Ibid).

Lo anterior no significa en absoluto que autoevaluación y acreditación tengan alguna incompatibilidad entre sí: la autoevaluación puede servir también al objetivo de acreditación, si la institución así lo considera dentro de su libre opción. Al respecto Ortiz Rozo anota:

"Otra cosa muy diferente es el que una vez que se haya ejecutado la autoevaluación con referencia al quehacer institucional se establezcan los mecanismos de articulación (o salidas) con los procesos de la heteroevaluación y la acreditación final". (Ibid)

Finalmente considerando que la acreditación es un acto de certificación pública respecto de la calidad de un servicio educativo, es claro que la autoevaluación constituye un requisito básico para los procesos de acreditación.

Es decir que la acreditación tiene como requisito básico la información generada por un proceso de autoevaluación (Cruz, 1991:6) pero la autoevaluación de ninguna manera debe tener como requisito el obtener la acreditación.

\subsection{Los factores culturales, económicos, bistóricos y políticos}

Aunque las universidades en todos los países comparten un "lenguaje" común internacional y diversos atributos de carácter general, las características principales de cualquier autoevaluación variarán de acuerdo con la influencia de las condiciones nacionales y locales. Sin ninguna duda el contexto influye sobre los propósitos de la evaluación y la naturaleza de estos debiera influir sobre los medios que se empleen en la autoevaluación.

Por ello el diseño de la autoevaluación debe estar articulado a las condiciones 
y necesidades de la institución de educación superior o programa objeto de la evaluación.

Ejemplo de factores que influyen en la autoevaluación son: (tomado de Kells, 1997: 24-28).

a. El poder relativo, conocimientos y actitudes que tengan los líderes.

b. Grado de desarrollo de una "cultura de autoevaluacioon".

c. Existencia de presión financiera. En todos los países trae como consecuencia un llamado a la eficiencia, a la competitividad, al uso de indicadores de desempeño y al incremento de capacidad para responder y reportar ante autoridades externas, entre otros.

d. Actitudes del gobierno. Estas influyen en la actividad evaluativa. Hay quienes manifiestan que el temor al control gubernamental es el que ha motivado la autorregulación en las universidades de norteamérica (Ibid:27).

\subsection{Condiciones para un proceso de autoevaluación}

Las siguientes son condiciones básicas para que se genere un proceso de autoevaluación; si estas no están dadas el equipo planificador debe accionar en estas direcciones.

\section{a. El apoyo de los lideres}

Se requiere motivación e interés de los líderes que asegure clima de confianza, continua prioridad del proceso, acceso a la información y otros recursos. Esta condición debe constituirse en un mensaje que se haga público en toda la institución o unidad. Algunas funciones vinculadas directamente con el ejecutivo en jefe en estos procesos son:

contribuir a formar la motivación interna hacia el proceso, asignarle una alta prioridad, apoyar al coordinador del estudio luego de haberse elegido como idóneo, esta responsabilidad la asume una co- misión elegida por la Asamblea de la Unidad Académica, proveer apoyo (recursos, otros) para el estudio,

ayudar o diseñar las estrategias para el cambio,

responder positivamente a formas nuevas de comportamiento que puedan ser generadas durante el estudio.

utilizar los resultados del estudio $\mathrm{y}$ promover la continuidad del proceso de autoevaluación y planeamiento.

\section{b. E1 conocimiento técnico experto}

El coordinador y miembros del grupo de planeamiento y conducción del proceso deben poseer un nivel adecuado de conocimiento técnico experto, métodos de evaluación. habilidades de grupo, métodos de recolección de datos y estrategias políticas y organizacionales para diseñar un proceso participativo comprometido por parte de los miembros de la institución o unidad. En el documento de Kells. $\mathbf{H}$. citado, se define como VI Etapa del proceso "La capacitación de los responsables del proceso" teniendo esta responsabilidad.

\section{c. Recursos}

Provisión adecuada de recursos tanto humanos como económicos para los procesos: el ejecutivo en jefe de la organización debe también considerar que se debe tenet alguna clase de garantía de que la institución implementará las recomendaciones razonables para el mejoramiento. La validación externa de las recomendaciones por parte de pares externos puede ser de gran ayuda en eso.

\section{d. Desarrollo de un adecuado grado de motivación interna}

Esta condición es de vital importancia. Los posibles participantes deben percibirlo como originado y efectuado en respuesta a las necesidades institucionales y no de una agencia externa. Esto debe constituirse en un mensaje permanente -continuidad de la persuación" (Kells, 1997:73) que debe ser enviado claramente por los líderes. 


\section{e. Una primera diagnosis de la agenda de problemas y necesidades locales}

La apreciación es una primera aproximación y da oportunidad a los participantes para hablar en términos útiles y generales acerca de las condiciones y del estado del desarrollo de la organización en discusión. No debe confundirse esto con el proceso detallado de estudio que vendrá a continuación.

\subsection{La participación: elemento central de la autoevaluación}

En la autoevaluación los distintos sectores involucrados se constituyen en sujetos interpretadores de sus acciones: detecta problemas, intercambia conocimientos y experiencias (comparaciones) y analizan y valoran el desarrollo de las actividades. Desde esta perspectiva el proceso de autoevaluación propuesto presenta las siguientes características:

a. focalizada: centrada en el análisis de los problemas,

b. diferenciada: genera diversidad de formas participativas en el proceso,

c. descentralizada: las decisiones dependen de los usuarios,

d. capacitación: en la acción: las interacciones enriquecen un proceso de capacitación recíproco,

e. intercomprensible: desarrolla creencias y significados comunes. La participación se construye en la confrontación de diferencias y contradicciones y posibilita entre otros, la creación e integración de conocimientos y experiencias.

\subsection{Aspectos operativos de la autoevaluación en instituciones de Educación Superior}

Algunos aspectos operativos son:

a. Respaldo institucional.

b. Propósito primario: el mejoramiento del desarrollo académico. Otros fines son: fomentar la planificación, fomentar la capacidad para diagnosticar fortalezas y debilidades en el ámbito interno y oportunidades y amenazas desde el entorno y buscar soluciones y estrategias que permitan sustentar los cambios necesarios.

c Énfasis: analítico orientado al cambio.

d. Referencias para los juicios evaluativos: la misión, políticos, fines y objetivos institucionales.

e. Validación externa: pares externos de reconocida excelencia.

f. Integración voluntaria.

g. Conducción: académicos de la propia institución o programa.

h. Carácter de aprendizaje continuo

i. Carácter secuencial: pueden ser ciclos de 4 a 6 años.

\subsection{Funciones básicas de la} autoevaluación institucional

Algunas funciones básicas son:

a. Función formativa, mejoramiento, corrección de errores.

b. Función sumativa, selección y responsabilidad, acumulación de puntos débiles que no pueden ser corregidos inmediatamente.

c. Función sociopolítica, motivación y ganar respaldo público.

d. Función administrativa, ejercicio de autoridad.

\section{La Autorregulación en la Educación Superior}

Según la Real Academia Española "regulación es un mecanismo que sirve para ordenar o normalizar el movimiento o los efectos de una máquina o de alguno de los órganos o piezas de ella" (Diccionario de la Lengua Española). En este sentido en las universidades públicas, que constituyen organismos sociales, obviamente también se hacen indispensables mecanismos para regular su funcionamiento. 
Tradicionalmente en las instituciones de Educación Superior en América Latina no han existido mecanismos fornales o explícitos de regulación. Han sido las propias instituciones las que han asumido esta tarea con mayor o menor participación del Estado. Fundamentalmente, la participación del último ha sido el aporte de los recursos financieros, sin interferir en sus decisiones de política ni en la evaluación de sus resultados.

Brunner y Briones; le llaman a esto "Coordinación corporativa con participación del Estado" (citado por Lemaitre M. J.: SF: 1).

A partir de la década de los 70's en América Latina se generó cada vez, mayor demanda por acceso a la Educación Superior, como consecuencia de la ampliación de la cobertura de los niveles de educación primaria y secundaria.

Los distintos países desarrollaron diferentes estrategias para enfrentar la mayor demanda. En general no se expanden las matrículas pero sí se produce la apertura de los sistemas proliferando las instituciones privadas en todos los países.

Aunado a la situación anterior la crisis económica internacional afecta cada vez con más rigor a la Educación Superior (desde el segundo quinquenio de la década del 70) presiona hacia la búsqueda de procesos de regulación conducentes hacia el mejoramiento óptimo en sus desempeños.

Los sistemas universitarios empiezan a sufrir modificaciones que hacen indispensable la regulación.

Obviamente lo que se necesita no es una regulación venida desde el Estado o desde el mercado. Respecto a lo último y refiriéndose a la situación de Chile Lemaitré M. J. anota: "El mercado como mecanismo regulador se ha demostrado ineficaz, y es preciso reconocerlo" (Ibid:6).

En la década de los 90's la mayoría de los países latinoamericanos se plantean el desafío de generar procesos de evaluación y regulación sistemáticos correspondientes a la acreditación "ideados" desde el "interior" de las instituciones. Kells, $H$. anota que "muchas instituciones en Canadá y Estados Unidos también están luchando por alejarse de los ejercicios evaluativos ad hoc (si bien algunos siempre serán necesarios) y de la dominancia de fuerzas y agencias externas en la evaluación" (Kells, 1997:39).

Al hacer referencia a esos retos pero en relación con la región centroamericana en las "Memorias del Primer Curso-taller sobre la Evaluación Superior" realizado en Guatemala, se plantea: "en sintesis el desafio puede resumirse en la necesidad y urgencia de construir un instrumento que contribuyera de manera significativa a la modernización de la Educación Superior Centroamericana y se constituya, de esta manera, en un instrumento de promoción y gestión del cambio, de la reforma universitaria (CSUCA, 1997:11).

Los procesos de autorregulación permiten a las instituciones de educación superior a partir de sí mismas, entender y mejorar el desempeño para responder a los retos que se afrontan: comprender fortalezas, problemas y oportunidades y diseñar estrategias para alcanzar metas y propósitos claramente definidos "es ella quien carga con la responsabilidad primaria por su excelencia y destino propios". (Kells, 1977:44). Es activo en lugar de ser solo reactivo a regulaciones emanadas del gobierno u otro externo.

Un ente autorregulado utiliza activamente un organismo de alto nivel u otro mecanismo para formular y sentar políticas referidas a actividades regulatorias, para ponderar resultados y para asignar algunos de los recursos marginales de la institución para costear las mejoras. Asigna responsabilidades a nivel ejecutivo y de personal para la actividad evaluativa ${ }^{3}$. (Ibid:45).

La socialización de los resultados (producto de la autoevaluación y autorregulación) genera compromiso e identidad institucional. Tal como se señaló antes: nada compromete más que el hecho de participar en la elaboración de las futuras modificaciones y actividades de mejoramiento.

Debido a que la calidad, asociada directamente a los procesos de autorregulación, es 
un ideal que no tiene fin, se requiere una permanente revisión del proceso. Los mejoramiento: deben ser procesos continuos y sostenidos porque existe la posibilidad de caer en la desidia y en la inercia. (Contreras B., 1995:11).

\section{Acreditación en la Educación Superior}

Agustín, J. y Miguel López definen la acreditación como un acto de reconocimiento público respecto de la calidad del servicio educativo.

Consideran los autores, que el aspecto medular de la acreditación es asegurar y estimular la calidad del servicio ofrecido: "asegurar a la sociedad y comunidad intelectual que se tiene solvencia académica". (citado por Ortiz Rozo, 1994:197)

Jacques L' ecuyer plantea que la acreditación es un medio para mejorar. Según "Manual de Autoevaluación para Instituciones de Educación Superior", la fe pública cautelada por la acreditación se establece en relación con los siguientes aspectos:

3

b La voluntad y solvencia para hacer lo que se promete.

- La coherencia entre procesos, misión y logros.

i. El establecimiento de estándares de calidad consistentes con la política de acreditación y,

- El mejoramiento contínuo mediante mecanismos adecuados (Espinoza, $\mathrm{O}$. 1994:45).

Por orro lado, Contreras Bello y otros zutore especifican un poco más su definixón de acreditación anotando que "la acredizaón es el reconocimiento público a la calitad de la educación que ofrece una universitad de acuerdo con su misión, sus objetivos y camateristicas especificas" (Contreras B., 19059.
El proceso de acreditación involucra y tiene como base la autoevaluación y autorregulación; esto en función de determinar el grado de cumplimiento de la misión, objetivos, el manejo de los recursos, la gestión en sí y la aproximación al deber ser que la misma institución se ha propuesto.

"La autoevaluación prepara a la institución para que pueda someterse a la mirada externa y a la acreditación que hace el Consejo Nacional de Acreditación". (Ibid:10)

\subsection{Noción de calidad en la Educación Superior}

Al considerar que acreditación es el reconocimiento público a la calidad de la educación superior que se ofrece, se hace necesario analizar, aunque sea de manera breve este concepto.

Tal como lo anotan Contreras Bello y otros, el concepto de calidad, en general, ha sido considerado una propiedad del acto educativo. El término en sí es intangible y relativo, por cuanto no puede ser en sí demostrado cuantitativo ni unilateralmente lo que significa que depende, en gran parte, del contexto, misión y visión de la institución educativa. Independiente de la posición que se adopte sobre las características de la acreditación (y en realidad cualquier modalidad de la función evaluativa) se hace indispensable "construir" una noción, modelo o esquema que describa qué se entiende por buena calidad de la Educación Superior. Debe adoptarse al respecto un punto de vista, aún a sabiendas de que nunca se habrá dicho la última palabra.

Según los autores arriba citados, los conceptos de excelencia y calidad son equivalentes: el primero busca optimizar recursos $\mathrm{y}$ actividades al interior de una preocupación constante por superarse-perfeccionarse.

Agregan estos autores que "la calidad se mira como el espacio de perfectibilidad que existe entre el deber ser y el ser de la institución, el cual exige un proceso de autoevaluación en función de un mejoramiento relacionado con los diferentes centros de atención. 
La calidad de la educación está ligada al concepto de formación integral del estudiante, del futuro profesional.

Para avanzar realmente en la calidad es preciso que la universidad ofrezca un servicio educativo relevante, eficiente y eficaz". (Ibid).

Según Gogo Huguet, los criterios de calidad de una institución o programa son:

Eficientes (medida en que se aprovechan los recursos disponibles).

Pertinentes (social, académica y científicamente).

Trascendente (en el tiempo, el espacio y otras tareas).

Equitativos (social, económica y pedagógicamente).

\subsection{Niveles de la acreditación}

La acreditación puede ser a nivel de toda la institución educativa o solo de programas: se sugiere comenzar por la acreditación de programas reconociendo que la acreditación institucional tiene exigencias de evaluación integral que van más allá de un simple agregado de la acreditación de programas o de bloques de programas". (ICFES. SF: 27):

La acreditación se inicia con la evaluación institucional la cual es continuada (revisada) por pares académicos de reconocida excelencia académica (que pueden ser sugeridos por la misma institución) y culmina con el acto de acreditación proferido por el órgano nacional instaurado para ese efecto. En Costa Rica en el año 1997 se creó para ese efecto el Sistema Nacional de Acreditación de la Educación (SINAES). En el caso de no obtenerse la acreditación en un marco de confidencialidad, se retroalimenta a la organización para que pueda subsanar debilidades y fallas. (Ibid).

Además de la autoevaluación institucional o de programas la acreditación involucra otros dos procesos evaluativos:

La heteroevaluación o evaluación por pares académicos sobre la calidad, que utiliza como punto de partida la autoevaluación. Consiste en identificar las condiciones internas de operación, verificar los resultados de la autoevaluación y concluye con un juicio sobre la calidad de la institución o programa que pretende ser acreditado.

- La evaluación síntesis realizada por el Consejo Nacional de Acreditación del país, sobre la base de la autoevaluación y de la heteroevaluación (Ibid:4).

\subsection{Caracteristicas de la Acreditación}

En la acreditación sobresalen dos características:

a. Su carácter temporal. Generalmente se confiere con una duración de 5 años. Se requiere una comprobación periódica ante pares académicos para continuar gozando de la acreditación.

b. Su carácter voluntario. El acogerme voluntariamente por parte de las instituciones implica que este es un proceso diferente al de inspección y vigilancia que debe ejercer el Estado, y que por tanto, la acreditación no reemplaza. (Ibid:26).

Al respecto Pedro Polo Verano expresa, "no ha de confundirse el sistema de acreditación (en Colombia) con los mecanismos de control que debe ejercer el Estado para proteger al público de la transgresión a las normas que rigen la educación superior" (Polo Verano. 1997:2).

En ese sentido lo que justifica la creación, en los países de un Sistema Nacional de Acreditación es el potencial que este tiene para un mejoramiento de las instituciones y de la educación superior en general, mayor que el que podrían lograr esas mismas instituciones de forma individual, aislada y espontánea (Ibid). Respecto a la propia responsabilidad que tienen las instituciones de mejorar su calidad, el último autor de origen colombiano manifiesta, "esta necesidad de 
asegurar la calidad en la educación superior, dentro de un régimen de autonomía universitaria. llevó al legislador a la concepción de un Sistema lacional de Acreditación, voluntario por ley. pero con la esperanza de que en la práctica todas las instituciones en el ejercicio responsable de su autonomía, lleguen al convencimiento de la necesidad de autoevaluarse como manifestación expresa de su compromiso con la calidad, y de abrir sus puertas al escrutinio de pares académicos y al dictamen final del Consejo Nacional de Acreditación" (Ibid).

\subsection{Otros aspectos que caracterizan la acreditación}

Proporciona la información necesaria y confiable para que se pueda dar a la sociedad la información básica sobre las instituciones que voluntariamente se sometieron a la acreditación. con el propósito de que el estudiante pueda tomar una mejor decisión acerca de instituciones y programas en los cuales puede adquirir su formación (ICFES. SF: 26).

La acreditación hace público el reconocimiento de las características sobresalientes de la institución y (o sus programas y de los logros alcanzados). (Ibid: 27).

\subsection{Modalidades de la acreditación}

La acreditación puede ser:
a) Institucional.
b) De Programas.
c) Internacional.

La primera, comprueba el desempeño institucional en relación a la misión; el segundo tipo de Acreditación (Programas) comprueba la calidad especialmente a los futuros. profesionales. También está la acreditación internacional.

Las decisiones sobre acreditación ajoptadas por el órgano correspondiente pueden ser
a) Acreditación sin condiciones.
b) Acreditación con informe de segui- miento.
c) Acreditación con visita especial.
d) Posponer la acreditación.

\subsection{Usos de la Acreditación}

a. Para identificar las instituciones educativas o programas con diferentes grados de excelencia.

b. Para desarrollar el principio de identidad institucional.

c. Para la realización de reconocimientos de estudios y traslados.

d. Para desarrollar la confiabilidad en los egresados.

\section{Conclusiones}

En los países latinoamericanos se escucha y se escribe cada vez con mayor frecuencia sobre los temas de acreditación de la educación superior. Sin embargo la mayoría de los documentos son de carácter conceptual y técnico y carecen la mayoría de estos, de análisis críticos que los ubiquen en la realidad latinoamericana. Ello evidencia también la ausencia en los procesos de acreditación - autoevaluación - autorregulación de variables centrales en el proceso educativo como son los fundamentos ético-epistemológico y enfoques pedagógicos.

Los tres conceptos establecen hoy, vinculaciones estrechas entre sí particularmente la autoevaluación y autorregulación. Ortiz Rozo inclusive, considera que la autoevaluación es en sí misma un mecanismo autorregulador.

Los distintos autores revisados asumen posiciones optimistas o positivas respecto a esos procesos. Consideran que por su mismo carácter voluntario y participativo permiten a las organizaciones de educación superior, generar respuestas prospectivas y no solo reactivas especialmente en estos tiempos de crisis económica y social. 
Respecto a las interacciones entre los procesos es oportuno insistir en la coincidencia de los autores respecto a la importancia de no supeditar el proceso de autoevaluación a la acreditación. Esta última es un requerimiento externo al quehacer y compromiso social de la institución de educación superior. Debido a que la acreditación supone un juicio, una certificación puede tender a distorcionar el proceso dë autoevaluación presentándose una imagen más ficticia que real. Sin embargo plantean los expertos revisados que lo anterior no significa en absoluto que autoevaluación y acreditación tengan alguna incompatibilidad entre sí: la autoevaluación puede servir también al objetivo de acreditación, si la institución así lo considera dentro de su libre opción.

Son procesos que actualmente se vinculan a los procesos de globalización y que por tanto, son de alguna manera inminentes aún no estando de acuerdo con ellos. Se debe revisar críticamente y asumir decisiones o al menos puntos de vista al respecto.

Los tres conceptos involucran procesos globales analíticos, reflexivos que tienen el propósito central de sustentar los cambios necesarios para mejorar el desempeño en la educación superior.

Se caracterizan por ser procesos voluntarios conducidos por las mismas personas involucradas en las instituciones o programas: los distintos sectores se constituyen en sujetos críticos interpretadores de sus acciones.

Fundamentalmente los autores latinoamericanos coinciden en que es necesario que estos procesos de autoevaluación y autorregulación estén articulados a las condiciones nacionales y necesidades específicas de las instituciones o programas que les dan origen.

\section{Notas}

1 Ello será objeto de un próximo artículo.

2 En el caso de una unidad académica al interior de una institución de educación superior, serian regulaciones procedentes de la jerarquía universitaria.

En Costa Rica se crea el Sistema Nacional de Acreditación de la Educación Superior (SINAES) que define las Comisiones de Acreditación nombrada por las Asambleas de las Unidades Académicas para que conduzcan estos procesos al interior de las unidades académicas. Este es un proceso total y voluntario de las Unidades Académicas.

\section{Referencias bibliográficas}

Agustín José y Miguel López. Asociación Dominicana para el Autoestudio y la Acreditación. Documento presentado al seminario sobre Acreditación Universitaria en América Latina, CINDA, Chile. 1993.

Castillo H., Mario y otros, Caracteristicas de Calidad: Hacia una Universidad Autorregulada. Colombia: Talleres Gráficos. Universidad de Antioquía. 1996.

CINDA. (s.f.). Autoevaluación. Chile.

Consejo Superior Universitario (CSUCA). Organización Universitaria Interamericana (OUI). Construyendo Criterios e Indicadores de Calidad para la Educación Superior en América Central. (Memoria del I Curso-taller Centroamericano sobre Evaluación y Acreditación de la Educación Superior). San José. 1997.

Contreras B., Yolanda y otros. Hacia una cultura del mejoramiento continuo. Documento mimeografiado. Universidad Jorge Tadeo Lozano. Bogotá, Colombia. 1995 .

Cruz Cardona, Víctor. Guia de Autoevaluación. España. Ediciones U.I.P. 1991. 
Espinoza, Oscar y otros. Manual Autoevaluación para instituciones de Educación Superior. Colombia. Edición CINDA/PROMESUP-OEA. 1994.

Gago Huguet, A. Calidad, Acreditación y Evaluación Institucional. Presentación en la reunión preparatoria a la Conferencia Regional. CRESAL-CSUCAANUIES, México. 1996.

Gurdián F., Alicia. Acreditación y Globalización. Memoria Seminario sobre Evaluación y Acreditación. San José. 1997.

ICFES. (s:f) Programa de Fomento a la Cultura de la Acreditación. Normas: Sistema Nacional de Acreditación. Colombia.

Kells, Herbert R. Procesos de Autoevaluación: una guía para la autoevaluación en la Educación Superior. Fondo Editorial de la Pontificia Universidad Católica dęl Perú. 1997.

L'ecuyer, Jacques. (s.f). Evaluación y Acreditación. Costa Rica: mimeografiado.

L'ecuyer, Jacques. La Evaluación y la Acreditación: medios para mejorar la calidad académica. San José. 1994.
Lemaitré, María José. (s.f). Desarrollo de un Sistema de Acreditación en Chile. Documento mimeografiado.

Ortiz Rozo, J. Alirio. Guía General para la Autoevaluación de la Universidad. Colombia. Edición Universidad INCCA. 1994.

Ortiz Rozo, J. Alirio. Trece reglas básicas para una autoevaluación. Seminario Internacional sobre autoevaluación y acreditación. Documento mimeografiado. Colombia. 1995.

Polo Verano, Pedro. El Sistema de Acreditación en Colombia. Presentación ante los participantes del primer curso taller organizado por el Consejo Superior Universitario Centroamericano. San José. 1997.

Real Academia Española. Diccionario de la Lengua Española. Vigésima Edición. España. 1984.

Silva T., M. (s.f). Sin título. Universidad de Concepción.

Masís, Carlos. Aportes para la reflexión del proceso de acreditación. Instituto Tecnológico de Costa Rica. 1997. 